\title{
BI-SPACES GLOBAL ATTRACTORS IN ABSTRACT PARABOLIC EQUATIONS
}

\author{
J. W. CHOLEWA and T. DŁOTKO \\ Institute of Mathematics, Silesian University \\ Bankowa 14, 40-007 Katowice, Poland \\ E-mail: jcholewa@ux2.math.us.edu.pl, tdlotko@ux2.math.us.edu.pl
}

\begin{abstract}
An abstract semilinear parabolic equation in a Banach space $X$ is considered. Under general assumptions on nonlinearity this problem is shown to generate a bounded dissipative semigroup on $X^{\alpha}$. This semigroup possesses an $\left(X^{\alpha}-Z\right)$-global attractor $\mathcal{A}$ that is closed, bounded, invariant in $X^{\alpha}$, and attracts bounded subsets of $X^{\alpha}$ in a 'weaker' topology of an auxiliary Banach space $Z$. The abstract approach is finally applied to the scalar parabolic equation in $R^{n}$ and to the partly dissipative system.
\end{abstract}

1. Introductory notes. The theory of global attractors was a very active field of studies through the last 20 years. One of the limitation inside the classical setting of this theory $([\mathrm{Ha}],[\mathrm{La}])$ was the requirement of compactness (or, at least, asymptotic compactness) of the semigroup in considered phase space. This assumption cannot be however satisfied when the space variable $x$ belongs to large unbounded domains, in particular, to the whole of $R^{n}$. To overcome this difficulty, it was necessary to generalize the idea of an attractor allowing the convergence to the attractor in a weaker topology than the topology of the phase space in which the semigroup acts. Such generalization was introduced in $[\mathrm{B}-\mathrm{V}]$ and further developed in $[\mathrm{M}]$ and $[\mathrm{M}-\mathrm{S}]$. We have already joined these studies in [Ch-Dl1] and [Ca-Dl]. The aim of the present note is to describe a general approach to such bi-spaces attractors for sectorial equations. We propose an abstract existence result (Theorem 2.6) and discuss its applications to two specific problems; bistable reaction-diffusion equation and FitzHugh-Nagumo system (Examples 3.1 and 3.6).

Both these problems are of special interest because of their nontrivial dynamics, which is partially connected with the existence of the families of travelling waves (or relaxation

Supported partially by the State Committee for Scientific Research (KBN) grant 2 P03A 03518.

2000 Mathematics Subject Classification: Primary 35B40, 35B41, 35K15, 35K45.

Key words and phrases: Parabolic equation, partly dissipative system, Cauchy problem, global solution, absorbing set, invariant attracting set.

The paper is in final form and no version of it will be published elsewhere. 
waves) convergent to constant equilibria. Mention should be made that the attractor we construct contains these special solutions as well. The latter was possible to achieve thanks to the specific function spaces involved in the considerations; namely locally uniform Sobolev spaces $W_{\mathrm{lu}}^{k, p}\left(R^{n}\right)$ (see Appendix). The idea of using such spaces in the studies of parabolic problems comes back to [M-S], [M], as well as to the earlier publication $[\mathrm{F}]$. A particular feature of the present paper is that it extends in a natural way the abstract results of $[\mathrm{Ch}-\mathrm{Dl}]$ concerning the existence of a global attractor to the case when the system is not even asymptotically compact.

2. Setting of the problem. Consider an abstract Cauchy problem

$$
\dot{u}+A u=F(u), t>0, \quad u(0)=u_{0},
$$

under the assumptions that

(i) $-A: X \supset D_{X} \rightarrow X$ generates a strongly continuous analytic semigroup in a Banach space $X$ and, for certain $\alpha \in[0,1), F: X^{\alpha} \rightarrow X$ is Lipschitz continuous on bounded sets (see $[\mathrm{He}]$ for the definition of fractional order space $X^{\alpha}$ ).

REMARK 2.1. As well known (see [He], [Ch-Dl]), the problem (1) is then locally well posed in $X^{\alpha}$ and it enjoys a solution

$$
u=u\left(\cdot, u_{0}\right) \in C\left(\left[0, \tau_{u_{0}}\right), X^{\alpha}\right) \cap C\left(\left(0, \tau_{u_{0}}\right), X^{1}\right) \cap C^{1}\left(\left(0, \tau_{u_{0}}\right), X^{1^{-}}\right),
$$

$\tau_{u_{0}}$ being a 'life time' of $u$.

Assume further that

(ii) the unique solution $u=u\left(\cdot, u_{0}\right), u_{0} \in X^{\alpha}$, exists globally in time,

(iii) the semigroup $\{T(t)\}$ of global solutions corresponding to (1) on $X^{\alpha}$ has bounded orbits of bounded sets and there exists a bounded set $B_{0} \subset X^{\alpha}$ absorbing bounded subsets of $X^{\alpha}$ under $\{T(t)\}$, that is:

$$
\forall B \text { bounded in } X^{\alpha} \exists t_{B} \geq 0 \bigcup_{t \geq t_{B}} T(t) B \subset B_{0} .
$$

REMARK 2.2. Note that (i) is the usual assumption to study sectorial evolutionary equation (see e.g. [Ha]). Concerning assumptions (ii)-(iii), one may refer to [Ch-Dl, Corollary 4.1.3], where conditions for the existence of corresponding to (1) bounded dissipative semigroup were established. Namely, the solutions should be a priori bounded in the norm of some auxiliary Banach space $Y, X^{1} \subset Y \subset X$,

$$
\left\|u\left(t, u_{0}\right)\right\|_{Y} \leq c\left(\left\|u_{0}\right\|_{X^{\alpha}}\right) .
$$

This bound should be asymptotically independent of initial condition varying in bounded subsets of $X^{\alpha}$. Simultaneously, the nonlinear term should be subordinated to $A^{\alpha}$ according to the condition

$$
\left\|F\left(u\left(t, u_{0}\right)\right)\right\|_{X} \leq g\left(\left\|u\left(t, u_{0}\right)\right\|_{Y}\right)\left(1+\left\|u\left(t, u_{0}\right)\right\|_{X^{\alpha}}^{\theta}\right),
$$

where $\theta \in[0,1)$ and $g$ is a nondecreasing function. 
We will next focus on the case when there exists another Banach space $Z$, such that

(iv) $X \subset Z$ algebraically and topologically and $-A$ with the domain $D_{Z}$ such that $D_{X} \subset D_{Z}$ generates a strongly continuous linear semigroup on $Z$,

(v) for each set $B$ bounded in $X^{\alpha}$ there exists a constant $L_{B}>0$, such that

$$
\|F(v)-F(w)\|_{Z} \leq L_{B}\|v-w\|_{Z}, v, w \in B .
$$

In the following lemma we will prove that the solutions to (1) are continuous with respect to the initial data in a specific way. Such property will be crucial in the proof of Theorem 2.6 below.

Lemma 2.3. Under the assumptions (i)-(v), if $\left\{u_{n}\right\}$ is bounded in $X^{\alpha}, v \in X^{\alpha}$ and $\left\|u_{n}-v\right\|_{Z} \rightarrow 0$, then

$$
\left\|u\left(t, u_{n}\right)-u(t, v)\right\|_{Z} \rightarrow 0 \text { for each } t>0 .
$$

Proof. Thanks to the variation of constants formula (assumptions (i), (ii)) we obtain that

$$
u\left(t, u_{n}\right)-u(t, v)=S_{X}(t)\left[u_{n}-v\right]+\int_{0}^{t} S_{X}(t-s)\left[F\left(u\left(s, u_{n}\right)\right)-F(u(s, v))\right] d s
$$

where $\left\{S_{X}(t)\right\}$ is an analytic semigroup corresponding to $-A$ on $X$. Writing the exponential formula we have

$$
\left\|S_{X}(t) x-\left(\operatorname{Id}+\frac{t}{n} A\right)^{-n} x\right\|_{X} \rightarrow 0 \text { for } x \in X,
$$

and, if $\left\{S_{Z}(t)\right\}$ denotes a $C^{0}$-semigroup generated by $-A$ on $Z$ (assumption (iv)), also

$$
\left\|S_{Z}(t) z-\left(\operatorname{Id}+\frac{t}{n} A\right)^{-n} z\right\|_{Z} \rightarrow 0 \text { for } z \in Z \text {. }
$$

Since $X \subset Z$, it is clear that

$$
S_{X}(t) x=S_{Z}(t) x \text { for } x \in X
$$

and thus (3) reads

$$
u\left(t, u_{n}\right)-u(t, v)=S_{Z}(t)\left[u_{n}-v\right]+\int_{0}^{t} S_{Z}(t-s)\left[F\left(u\left(s, u_{n}\right)\right)-F(u(s, v))\right] d s .
$$

Let $B \subset X^{\alpha}$ be a bounded set such that $v, u_{n} \in B, n \in N$. Estimating in the usual way (see $[\mathrm{He}]$ ), thanks to assumptions (iii) and (v) we get

$$
\begin{aligned}
& \left\|u\left(t, u_{n}\right)-u(t, v)\right\|_{Z} \leq M e^{\omega t}\left\|u_{n}-v\right\|_{Z} \\
& \quad+\int_{0}^{t} M e^{\omega(t-s)}\left\|F\left(u\left(s, u_{n}\right)\right)-F(u(s, v))\right\|_{Z} d s \\
& \leq c(T, \omega, M)\left\|u_{n}-v\right\|_{Z}+c(T, \omega, M) L_{B} \int_{0}^{t}\left\|u\left(s, u_{n}\right)-u(s, v)\right\|_{Z} d s,
\end{aligned}
$$

for $0<t<T<\infty$. Therefore, by the Gronwall inequality,

$$
\left\|u\left(t, u_{n}\right)-u(t, v)\right\|_{Z} \leq c(T, \omega, M)\left\|u_{n}-v\right\|_{Z} e^{c(T, \omega, M) L_{B} t}, 0<t<T<\infty,
$$

which completes the proof. 
Concerning the absorbing set $B_{0}$ (assumption (iii)) we observe below that, without loss of generality, $B_{0}$ may be assumed positively invariant and bounded in $X^{1}$ norm.

LEMMA 2.4. Under the assumptions (i)-(iii) there exists a positively invariant absorbing set, which is bounded in $X^{1}$.

Proof. It is easy to see that if (iii) holds then a positive orbit $\gamma^{+}\left(B_{0}\right)$ is bounded in $X^{\alpha}$ and absorbs bounded subsets of $X^{\alpha}$. The same is true for any image $T(t) \gamma^{+}\left(B_{0}\right)$ where $t>0$. Since there exists $t>0$ such that $T(t) \gamma^{+}\left(B_{0}\right)$ is bounded in $X^{1}$ (see [Ch-Dl, Lemma 3.2.1]), the proof is complete.

REMARK 2.5. Lemma 2.4 combined with Remark 2.2 shows an advantage of studying the sectorial equation (1). Some a priori estimate in $Y$ norm, which may be relatively weak and easy to obtain, implies the existence of an absorbing set bounded in much stronger $X^{1}$ norm (for example $Y=L_{\mathrm{lu}}^{p}\left(R^{n}\right)$ and $X^{1}=W_{\mathrm{lu}}^{2, p}\left(R^{n}\right)$ in the case of the scalar reaction diffusion equation (5)).

We are now ready to formulate an abstract result, which is a counterpart of [Ca-Dl, Theorem 1] in the case of sectorial equation (1).

TheOrEm 2.6. Let (i)-(v) hold and let $r:=\sup _{\phi \in \gamma^{+}\left(B_{0}\right)}\|\phi\|_{X^{\alpha}}$. Suppose also that

(vi) if $t_{n} \rightarrow \infty$ and $\left\{u_{n}\right\}$ is bounded in $X^{\alpha}$, then a sequence $\left\{T\left(t_{n}\right) u_{n}\right\}$ contains a subsequence $\left\{T\left(t_{n_{k}}\right) u_{n_{k}}\right\}$ convergent in $Z$ to certain $w \in B_{X^{\alpha}}(r)$, where $B_{X^{\alpha}}(r)$ is a closed ball in $X^{\alpha}$ of radius $r$ centered at zero.

Then, the semigroup $\{T(t)\}$ corresponding to (1) on $X^{\alpha}$ possesses a nonempty closed bounded invariant set $\mathcal{A} \subset X^{\alpha}$, being the union of bounded (in $X^{\alpha}$ ) invariant complete orbits of points. Furthermore, $\mathcal{A}$ is bounded in $X^{1}$, compact in $Z$, and it attracts bounded subsets of $X^{\alpha}$ in the topology of $Z$.

Proof. Define

$\mathcal{A}:=\left\{v \in B_{X^{\alpha}}(r):\left\|T\left(t_{n}\right) u_{n}-v\right\|_{Z} \rightarrow 0\right.$ for certain $\left\{u_{n}\right\} \subset B_{0}$ and $\left.t_{n} \rightarrow \infty\right\}$.

Needless to say that $\mathcal{A}$ is bounded in $X^{\alpha}$. Also, if $\left\{u_{n}\right\} \subset B_{0}$ and $t_{n} \rightarrow \infty$ then, as a consequence of (vi), $\left\{T\left(t_{n}\right) u_{n}\right\}$ possesses a subsequence $\left\{T\left(t_{n_{k}}\right) u_{n_{k}}\right\}$ convergent to certain $v \in B_{X^{\alpha}}(r)$. Therefore, $\mathcal{A}$ is nonempty.

Take further $v \in \mathcal{A}$ and $\left\{u_{n}\right\} \subset B_{0}, t_{n} \rightarrow \infty$ such that $\left\|T\left(t_{n}\right) u_{n}-v\right\|_{Z} \rightarrow 0$. Then $\left\|T\left(t+t_{n}\right) u_{n}-T(t) v\right\|_{Z} \rightarrow 0$ as a result of Lemma 2.3 and, according to (vi), $\left\{T\left(t+t_{n}\right) u_{n}\right\}$ has a subsequence convergent in $Z$ to certain $w \in B_{X^{\alpha}}(r)$. As a consequence, we have that $w=T(t) v$, which proves that $T(t) v \in \mathcal{A}$. We next consider a sequence $\left\{T\left(t_{n}-t\right) u_{n}\right\}$, which (via (vi)) has a subsequence $\left\{T\left(t_{n_{k}}-t\right) u_{n_{k}}\right\}$ convergent in $Z$ to certain $w \in \mathcal{A}$. Since Lemma 2.3 implies that $\left\|T\left(t_{n_{k}}\right) u_{n_{k}}-T(t) w\right\|_{Z} \rightarrow 0$ (recall that, by assumption (iii), a positive orbit $\gamma^{+}\left(\left\{u_{n}\right\}\right)$ is bounded in $\left.X^{\alpha}\right)$ we obtain $v=T(t) w$. This justifies the invariance of $\mathcal{A}$.

To prove that $\operatorname{cl}_{X^{\alpha}} \mathcal{A}=\mathcal{A}$ take $\left\{v_{n}\right\} \subset \mathcal{A}$ and $w \in \operatorname{cl}_{X^{\alpha}} \mathcal{A}$ such that $\left\|v_{n}-w\right\|_{X^{\alpha}} \rightarrow 0$. Since $X^{\alpha} \subset X \subset Z$, we have $\left\|v_{n}-w\right\|_{Z} \rightarrow 0$ and, by definition of $\mathcal{A}$, there exist $\left\{u_{n}\right\} \subset B_{0}$, $t_{n} \rightarrow \infty$ for which $\left\|v_{n}-T\left(t_{n}\right) u_{n}\right\|_{Z} \rightarrow 0$. Recalling definition of $\mathcal{A}$ and assumption (vi) we conclude that $w \in \mathcal{A}$. 
Boundedness of $\mathcal{A}$ in $X^{1}$ is a consequence of the invariance of $\mathcal{A}$ and the arguments used in the proof of Lemma 2.4.

Based on assumption (vi) we next observe that $\mathcal{A}$ is compact in $Z$. Consider $\left\{v_{n}\right\} \subset \mathcal{A}$ and $\left\{u_{n}\right\} \subset B_{0}, t_{n} \rightarrow \infty$ such that $\left\|v_{n}-T\left(t_{n}\right) u_{n}\right\|_{Z} \rightarrow 0$. Condition (vi) ensures then the existence of certain $w \in B_{X^{\alpha}}(r)$ such that $\left\|w-T\left(t_{n_{k}}\right) u_{n_{k}}\right\|_{Z} \rightarrow 0$. Clearly $w \in \mathcal{A}$ and $\left\|v_{n_{k}}-w\right\|_{Z} \rightarrow 0$, which ensures compactness of $\mathcal{A}$ in $Z$.

It is now easy to see that if $B$ is bounded in $X^{\alpha}$, then

$$
\sup _{u \in B} \inf _{v \in \mathcal{A}}\|T(t) u-v\|_{Z} \rightarrow 0 \text { as } t \rightarrow \infty .
$$

Indeed, if it was not the case, we could choose $t_{n} \rightarrow \infty$ and $\left\{u_{n}\right\} \subset B$ such that

$$
\inf _{v \in \mathcal{A}}\left\|T\left(t_{n}\right) u_{n}-v\right\|_{Z}>\varepsilon
$$

for certain $\varepsilon>0$. However, since (iii) and (vi) hold, the latter condition is impossible.

Finally denote by $\mathcal{U}$ a union of bounded in $X^{\alpha}$, invariant, complete orbits of points. By invariance of $\mathcal{A}$ we observe that $\mathcal{A} \subset \mathcal{U}$. Also, if $\gamma\left(u_{0}\right)=\gamma^{-}\left(u_{0}\right) \cup \gamma^{+}\left(u_{0}\right)$ is such an orbit (see e.g. [Ch-Dl, p. 3]), then

$$
\sup _{u \in \gamma\left(u_{0}\right)} \inf _{v \in \mathcal{A}}\|u-v\|_{Z}=0
$$

which (by closeness of $\mathcal{A}$ in $Z$ ) ensures that $\gamma\left(u_{0}\right) \subset \mathcal{A}$.

The proof of Theorem 2.6 is thus complete.

REMARK 2.7. Assumption (vi) provides us some sort of asymptotic compactness. Despite certain complexity of the formulation, in applications condition (vi) is just a consequence of the Sobolev embeddings between appropriately chosen function spaces (see Section 3 below).

Recall that

Definition 2.8. A set $\mathcal{A} \subset X^{\alpha}$, which is invariant, closed in $X^{\alpha}$, compact in $Z$ and attracts bounded subsets of $X^{\alpha}$ in the topology of $Z$ is called an $\left(X^{\alpha}-Z\right)$-global attractor.

Therefore, Theorem 2.6 provides us the existence of an $\left(X^{\alpha}-Z\right)$-global attractor for (1), which is additionally bounded in the norm of $X^{1}$.

\section{Applications}

EXAmple 3.1. Our first example is a scalar reaction diffusion equation

$$
u_{t}=\Delta u+u\left(1-|u|^{q-1}\right), t>0, x \in R^{n},
$$

with arbitrarily fixed $q \in(1, \infty)$.

Let us specify in this example: $X=L_{\mathrm{lu}}^{p}\left(R^{n}\right), D_{X}=X^{1}=W_{\mathrm{lu}}^{2, p}\left(R^{n}\right), p>n, \alpha=\frac{1}{2}$, $X^{1 / 2}=W_{\mathrm{lu}}^{1, p}\left(R^{n}\right), Z=L_{\rho}^{p}\left(R^{n}\right), D_{Z}=W_{\rho}^{2, p}\left(R^{n}\right), f(u)=u\left(1-|u|^{q-1}\right)$ and $F(\phi)(x)=$ $f(\phi(x)), \phi \in W_{\mathrm{lu}}^{1, p}\left(R^{n}\right)$. For the definitions of these spaces see the Appendix.

As reported in $[\mathrm{M}]$ and $[\mathrm{Ch}-\mathrm{Dl} 1],-\Delta$ is a densely defined sectorial operator both in $L_{\mathrm{lu}}^{p}\left(R^{n}\right)$ and in $L_{\rho}^{p}\left(R^{n}\right)$. Also, $W_{\mathrm{lu}}^{1, p}\left(R^{n}\right) \subset C_{\mathrm{bd}}^{0}\left(R^{n}\right)$ (see [Ch-Dl1]). Therefore, if $B$ is 
bounded in $W_{\text {lu }}^{1, p}\left(R^{n}\right)$ and $v, w \in B$, then values $v(x), w(x)\left(x \in R^{n}\right)$ vary in a bounded subset $I \subset R^{1}$. Since $f: I \rightarrow R^{1}$ is globally Lipschitz, we have

$$
\begin{aligned}
& \|F(v)-F(w)\|_{X}=\sup _{y \in R^{n}}\left(\int_{R^{n}}|f(v(x))-f(w(x))|^{p} \rho(x-y) d x\right)^{1 / p} \\
& \leq \sup _{y \in R^{n}}\left(\int_{R^{n}} L_{B}^{p}|v(x)-w(x)|^{p} \rho(x-y) d x\right)^{1 / p}=L_{B}\|v-w\|_{L_{\mathrm{lu}}^{p}\left(R^{n}\right)},
\end{aligned}
$$

as well as

$$
\begin{aligned}
& \|F(v)-F(w)\|_{Z}=\left(\int_{R^{n}}|f(v(x))-f(w(x))|^{p} \rho(x) d x\right)^{1 / p} \\
& \quad \leq\left(\int_{R^{n}} L_{B}|v(x)-w(x)|^{p} \rho(x) d x\right)^{1 / p}=L_{B}\|v-w\|_{Z} .
\end{aligned}
$$

The above considerations show that assumptions (i), (iv) and (v) are satisfied in this example.

To check validity of (ii) and (iii) we first obtain a subordination condition (2) (with $\left.Y=L_{\mathrm{lu}}^{p q}\left(R^{n}\right)\right)$ having the form

$$
\begin{aligned}
\left\|F\left(u\left(t, u_{0}\right)\right)\right\|_{L_{\mathrm{lu}}^{p}\left(R^{n}\right)} & =\sup _{y \in R^{n}}\left(\int_{R^{n}}\left|u\left(t, u_{0}\right)\left(1-\left|u\left(t, u_{0}\right)\right|^{q-1}\right)\right|^{p} \rho(x-y) d x\right)^{1 / p} \\
\leq & \text { const. }\left(1+\left\|u\left(t, u_{0}\right)\right\|_{L_{\mathrm{lu}}^{p q}\left(R^{n}\right)}^{q}\right)=: g\left(\left\|u\left(t, u_{0}\right)\right\|_{L_{\mathrm{lu}}^{p q}\left(R^{n}\right)}\right) .
\end{aligned}
$$

Now, as it is mentioned in Remark 2.2, we need merely find an appropriate $L_{\text {lu }}^{p q}\left(R^{n}\right)$ estimate for a solution $u\left(\cdot, u_{0}\right)$ of (5) with initial condition $u_{0} \in W_{\text {lu }}^{1, p}\left(R^{n}\right)$. This will be done in lemma below.

Lemma 3.2. Let $B \subset W_{\mathrm{lu}}^{1, p}\left(R^{n}\right)$ be a bounded set and $s \geq 2$. Then, solutions to (5) fulfil the estimates

$$
\begin{gathered}
\sup _{u_{0} \in B}\left\|u\left(t, u_{0}\right)\right\|_{L_{1 \mathrm{u}}^{s}\left(R^{n}\right)} \leq \sup _{u_{0} \in B}\left\|u_{0}\right\|_{L_{\mathrm{lu}}^{s}\left(R^{n}\right)}+c_{s}, \\
\limsup _{t \rightarrow+\infty}\left(\sup _{u_{0} \in B}\left\|u\left(t, u_{0}\right)\right\|_{L_{\mathrm{lu}}^{s}\left(R^{n}\right)}\right) \leq c_{s} .
\end{gathered}
$$

Proof. Multiplying (5) by $u|u|^{s-2} \rho(x-y)$ and integrating, we find

$$
\begin{aligned}
\frac{1}{s} \frac{d}{d t} \int_{R^{n}}|u|^{s} \rho(x-y) d x=\int_{R^{n}} \Delta u u|u|^{s-2} \rho(x-y) d x & \\
& +\int_{R^{n}}\left(1-|u|^{q-1}\right)|u|^{s} \rho(x-y) d x .
\end{aligned}
$$

Since $|\nabla \rho(x-y)| \leq c_{0} \varepsilon \rho(x-y)$ (see $\left.(26)\right)$, we have

$$
\begin{array}{r}
\int_{R^{n}} \Delta u u|u|^{s-2} \rho(x-y) d x \leq \int_{R^{n}}|u|^{s-2}\left(-(s-1)|\nabla u|^{2}+c_{0} \varepsilon|\nabla u||u|\right) \rho(x-y) d x \\
\leq \frac{1}{4(s-1)} c_{0}^{2} \varepsilon^{2} \int_{R^{n}}|u|^{s} \rho(x-y) d x
\end{array}
$$


and

$$
\int_{R^{n}}\left(1-|u|^{q-1}\right)|u|^{s} \rho(x-y) d x \leq-\int_{R^{n}}|u|^{s} \rho(x-y) d x+M_{s},
$$

where $M_{s}:=\sup _{|u|^{q-1}<2}\left(\left.\left.|1-| u\right|^{q-1}|| u\right|^{s}\right) \int_{R^{n}} \rho(x-y) d x$. Collecting the above estimates we obtain

$$
\frac{1}{s} \frac{d}{d t} \int_{R^{n}}|u|^{s} \rho(x-y) d x \leq\left(\frac{c_{0}^{2} \varepsilon^{2}}{4(s-1)}-1\right) \int_{R^{n}}|u|^{s} \rho(x-y) d x+M_{s},
$$

which for $\varepsilon$ small enough, such that $-m_{s}:=\frac{c_{0}^{2} \varepsilon^{2}}{4(s-1)}-1<0$, gives an estimate

$$
\int_{R^{n}}|u|^{s} \rho(x-y) d x \leq\left(\int_{R^{n}}\left|u_{0}\right|^{s} \rho(x-y) d x\right) e^{-s m_{s} t}+\frac{M_{s}}{m_{s}}\left(1-e^{-s m_{s} t}\right),
$$

leading to $(7)$ and (8).

What was said above ensures that equation (5) generates on $W_{\mathrm{lu}}^{1, p}\left(R^{n}\right)$ a $C^{0}$-semigroup of global solutions which has bounded orbits of bounded sets and possesses a bounded absorbing set $B_{0}$. Let us remark that, thanks to Lemma 2.4, we may assume without loss of generality that $B_{0}$ is positively invariant and bounded in $W_{\mathrm{lu}}^{2, p}\left(R^{n}\right)$.

Concerning validity of (vi) we will show further that this condition follows from the embedding of locally uniform spaces into Hölder spaces. Indeed, let $B_{W_{\mathrm{lu}}^{1, p}\left(R^{n}\right)}(r)$ be a closed ball containing $B_{0}$ and choose a sequence $\left\{u_{n}\right\}$ bounded in $W_{\mathrm{lu}}^{1, p}\left(R^{n}\right)$ and $t_{n} \rightarrow \infty$. Since $\left\{u_{n}\right\}$ is absorbed by $B_{0}$ and $B_{0}$ is bounded in $W_{\text {lu }}^{2, p}\left(R^{n}\right)$, we observe that $\left\{T\left(t_{n}\right) u_{n}, n \geq n_{0}\right\}$ is bounded in $W_{\text {lu }}^{2, p}\left(R^{n}\right)$ and we define $v_{n}:=T\left(t_{n+n_{0}}\right) u_{n+n_{0}}, n \in N$.

We will prove that

LEMMA 3.3. The sequence $\left\{v_{n}\right\}$ bounded in $W_{\mathrm{lu}}^{2, p}\left(R^{n}\right)$ contains a subsequence convergent in $L_{\rho}^{p}\left(R^{n}\right)$ to an element $v \in W_{\text {lu }}^{1, p}\left(R^{n}\right)$.

Proof. As a consequence of Lemma 4.2, for fixed $\mu \in\left(0,1-\frac{n}{p}\right), W_{\mathrm{lu}}^{2, p}\left(R^{n}\right) \subset$ $C^{1+\mu}\left(R^{n}\right)$. Hence $\left\{v_{n}\right\}$ is bounded in $C^{1+\mu}\left(R^{n}\right)$, which implies that $\left\{v_{n}\right\}$ is precompact in $C^{1+\nu}(\{|x| \leq k\})$ for each $k \in N$ and arbitrarily fixed $\nu \in(0, \mu)$ (see [Ad, Theorem 1.31]). The latter ensures the existence of a (diagonal) subsequence $\left\{v_{n_{k}}\right\}$ convergent in each space $C^{1+\nu}(\{|x| \leq k\}), k \in N$, to certain $v \in C^{1+\nu}\left(R^{n}\right)$. Evidently, $\left\|v_{n_{k}}-v\right\|_{L_{\rho}^{p}\left(R^{n}\right)} \rightarrow 0$ as a result of Lebesgue dominant convergence theorem and we will show below that such $v$ belongs to $W_{\text {lu }}^{1, p}\left(R^{n}\right)$.

Since function $v$ is uniformly bounded on $R^{n}$ together with the first order derivatives we only need to justify translation continuity property

$$
\left\|\tau_{z} v-v\right\|_{W_{\mathrm{lu}}^{1, p}\left(R^{n}\right)} \rightarrow 0 \text { as }|z| \rightarrow 0
$$

where $\tau_{z} v(x)=v(x-z), x, z \in R^{n}$. We start with an estimate

$$
\begin{aligned}
& \left(\int_{R^{n}}\left|v_{x_{k}}(x-z)-v_{x_{k}}(x)\right|^{p} \rho(x-y) d x\right)^{1 / p} \\
& \leq\left(\int_{R^{n}}\left|v_{x_{k}}(x-z)-v_{n x_{k}}(x-z)\right|^{p} \rho(x-y) d x\right)^{1 / p} \\
& \quad+|z|^{\nu}\left\|v_{n x_{k}}\right\|_{C^{\nu}\left(R^{n}\right)}\left(\int_{R^{n}} \rho(x-z) d x\right)^{1 / p}+\left(\int_{R^{n}}\left|v_{n x_{k}}(x)-v_{x_{k}}(x)\right|^{p} \rho(x-y) d x\right)^{1 / p}
\end{aligned}
$$


and pass to a lower limit as $k \rightarrow \infty$ to get

$$
\left(\int_{R^{n}}\left|v_{x_{k}}(x-z)-v_{x_{k}}(x)\right|^{p} \rho(x-y) d x\right)^{1 / p} \leq C|z|^{\nu}
$$

where $C:=$ const. $\sup \left\{\left\|v_{n}\right\|_{W_{\mathrm{lu}}^{2, p}\left(R^{n}\right)}, n \in N\right\}$. Similarly we show that

$$
\left(\int_{R^{n}}|v(x-z)-v(x)|^{p} \rho(x-y) d x\right)^{1 / p} \leq C|z|^{\nu},
$$

which together with (10) justifies validity of (9).

REMARK 3.4. Note that additionally,

$$
\begin{aligned}
& \left(\int_{R^{n}}|v(x)|^{p} \rho(x-y) d x\right)^{1 / p}+\sum_{k=1}^{n}\left(\int_{R^{n}}\left|v_{x_{k}}(x)\right|^{p} \rho(x-y) d x\right)^{1 / p} \\
& \quad \leq \sup _{n \in N} \sup _{y \in R^{n}}\left(\left(\int_{R^{n}}\left|v_{n}(x)\right|^{p} \rho(x-y) d x\right)^{1 / p}+\sum_{k=1}^{n}\left(\int_{R^{n}}\left|v_{n x_{k}}(x)\right|^{p} \rho(x-y) d x\right)^{1 / p}\right) \\
& +\left(\int_{R^{n}}\left|v(x)-v_{n}(x)\right|^{p} \rho(x-y) d x\right)^{1 / p}+\sum_{k=1}^{n}\left(\int_{R^{n}}\left|v_{x_{k}}(x)-v_{n x_{k}}(x)\right|^{p} \rho(x-y) d x\right)^{1 / p} .
\end{aligned}
$$

In particular, since $\left\{v_{n}\right\} \subset B_{W_{\mathrm{lu}}^{1, p}\left(R^{n}\right)}(r)$ and $v_{n} \rightarrow v$ (together with first order derivatives) uniformly on each compact subset of $R^{n}$, passing to a limit in the above inequality we obtain that

$$
\left(\int_{R^{n}}|v(x)|^{p} \rho(x-y) d x\right)^{1 / p}+\sum_{k=1}^{n}\left(\int_{R^{n}}\left|v_{x_{k}}(x)\right|^{p} \rho(x-y) d x\right)^{1 / p} \leq r .
$$

Adding supremum on the left hand side of (11), we justify that $v \in B_{W_{1 \mathrm{u}}^{1, p}\left(R^{n}\right)}(r)$.

Corollary 3.5. For $p>n$, the $C^{0}$-semigroup defined by $(5)$ on $W_{\mathrm{lu}}^{1, p}\left(R^{n}\right)$ possesses $a\left(W_{\mathrm{lu}}^{1, p}\left(R^{n}\right)-L_{\rho}^{p}\left(R^{n}\right)\right)$-global attractor, which is bounded in $W_{\mathrm{lu}}^{2, p}\left(R^{n}\right)$.

ExAmple 3.6. Our second example will be the FitzHugh-Nagumo system in $R^{n}$, $n \leq 4$,

$$
\left\{\begin{array}{l}
u_{t}=\Delta u-\alpha v+f(u), \quad t>0, x \in R^{n}, \\
v_{t}=-\delta v+\beta u+h(x), \\
u(0)=u_{0}, v(0)=v_{0},
\end{array}\right.
$$

where $\alpha, \beta, \delta$ are positive constants, $h \in L_{\text {lu }}^{p_{0}}\left(R^{n}\right)$ for certain $p_{0}>n$ and $f: R^{1} \rightarrow R^{1}$ is a locally Lipschitz continuous function satisfying

$$
f(s) s \leq-a s^{2}+b, s \in R^{1}, a, b>0 .
$$

For simplicity of the presentation we will restrict our further studies to the polynomially bounded nonlinearity

$$
|f(s)| \leq C\left(1+|s|^{z}\right), z>1 \text { arbitrary, } s \in R^{1} .
$$


System (12) will be rewritten in a matrix form

$$
\left(\begin{array}{l}
u_{t} \\
v_{t}
\end{array}\right)=\left(\begin{array}{cc}
\Delta & 0 \\
0 & -\delta
\end{array}\right)\left(\begin{array}{l}
u \\
v
\end{array}\right)+\left(\begin{array}{c}
f(u)-\alpha v \\
\beta u+h(x)
\end{array}\right)
$$

and considered in the space $X=L_{\mathrm{lu}}^{p}\left(R^{n}\right) \times L_{\mathrm{lu}}^{p}\left(R^{n}\right)$ where $n<p \leq p_{0}$. The operator $A=$ $-\Delta \times \delta$ defined on $D_{X}=W_{\mathrm{lu}}^{2, p}\left(R^{n}\right) \times L_{\mathrm{lu}}^{p}\left(R^{n}\right)$, is sectorial in $L_{\mathrm{lu}}^{p}\left(R^{n}\right) \times L_{\mathrm{lu}}^{p}\left(R^{n}\right)$. Thanks to the inclusion $X^{1 / 2}=W_{\mathrm{lu}}^{1, p}\left(R^{n}\right) \times L_{\mathrm{lu}}^{p}\left(R^{n}\right) \subset C_{\mathrm{bd}}^{0}\left(R^{n}\right) \times L_{\mathrm{lu}}^{p}\left(R^{n}\right)$, it is easy to verify Lipschitz continuity of the nonlinear term $F(\phi, \psi)(x)=(f(\phi(x))-\alpha \psi(x), \beta \phi(x)+h(x))$ acting from bounded subsets of $X^{1 / 2}$ into $X$. Therefore, (i) holds.

To show global solvability condition (ii), we need to obtain $L_{\mathrm{lu}}^{p z}\left(R^{n}\right) \times L_{\mathrm{lu}}^{p}\left(R^{n}\right)$ a priori estimate of the solution $(u, v)$.

Step 1. First the $L_{\mathrm{lu}}^{2}\left(R^{n}\right) \times L_{\mathrm{lu}}^{2}\left(R^{n}\right)$ estimate will be obtained. Multiplying the first equation in (12) by $\beta u \rho(x-y)$, the second by $\alpha v \rho(x-y)$, integrating and adding the results, we get

$$
\begin{aligned}
\frac{1}{2} \frac{d}{d t} \int_{R^{n}}\left(\beta|u|^{2}+\alpha|v|^{2}\right) \rho(x-y) d x+\beta \int_{R^{n}}|\nabla u|^{2} \rho(x-y) d x \\
=-\beta \int_{R^{n}} u \nabla u \cdot \nabla \rho(x-y) d x-\alpha \delta \int_{R^{n}} v^{2} \rho(x-y) d x \\
\quad+\beta \int_{R^{n}} f(u) u \rho(x-y) d x+\alpha \int_{R^{n}} h(x) v \rho(x-y) d x .
\end{aligned}
$$

Now, the estimate $|\nabla \rho(x-y)| \leq c_{0} \varepsilon \rho(x-y)$ (see (26)), condition (13), and the standard use of the Cauchy inequality gives

$$
\begin{aligned}
& \frac{d}{d t} \int_{R^{n}}\left(\beta|u|^{2}+\alpha|v|^{2}\right) \rho(x-y) d x \leq-\alpha \delta \int_{R^{n}} v^{2} \rho(x-y) d x \\
& \quad-a \beta \int_{R^{n}} u^{2} \rho(x-y) d x+2 b \beta \int_{R^{n}} \rho(x-y) d x+\frac{\alpha}{\delta} \int_{R^{n}} h^{2}(x) \rho(x-y) d x .
\end{aligned}
$$

Consequently, whenever $\left\|\left(u_{0}, v_{0}\right)\right\|_{L_{\mathrm{lu}}^{2}\left(R^{n}\right) \times L_{\mathrm{lu}}^{2}\left(R^{n}\right)} \leq \lambda$, we have

$$
\sup _{y \in R^{n}} \int_{R^{n}}\left(|u(t)|^{2}+|v(t)|^{2}\right) \rho(x-y) d x \leq C\left(\lambda,\|h\|_{L_{\mathrm{lu}}^{2}\left(R^{n}\right)}\right)
$$

and, asymptotically,

$$
\begin{aligned}
\limsup \sup _{t \rightarrow \infty} \int_{y \in R^{n}}\left(\beta|u(t)|^{2}\right. & \left.+\alpha|v(t)|^{2}\right) \rho(x-y) d x \\
& \leq \frac{1}{\min \{a, \delta\}}\left(2 b \beta \int_{R^{n}} \rho(x) d x+\frac{\alpha}{\delta}\|h\|_{L_{\mathrm{lu}}^{2}\left(R^{n}\right)}^{2}\right) .
\end{aligned}
$$

Step 2. We will next use (17) to obtain $L_{\mathrm{lu}}^{q}\left(R^{n}\right)$ estimate of $u$ with $q:=p z$. Without loss of generality we will assume that $q \geq 2$. 
Multiply the first equation in (12) by $u|u|^{q-2} \rho(x-y)$ and integrate over $R^{n}$ to get

$$
\begin{aligned}
\frac{1}{q} \frac{d}{d t} \int_{R^{n}}|u|^{q} \rho(x-y) d x & =\int_{R^{n}} \Delta u u|u|^{q-2} \rho(x-y) d x \\
& -\int_{R^{n}} \alpha v u|u|^{q-2} \rho(x-y) d x+\int_{R^{n}} f(u) u|u|^{q-2} \rho(x-y) d x
\end{aligned}
$$

Recalling (13), observing that $|\nabla u|^{2}|u|^{q-2}=\frac{4}{q^{2}}\left|\nabla\left(|u|^{q / 2}\right)\right|^{2}$, and keeping in mind that $L_{\mathrm{lu}}^{2}\left(R^{n}\right)$ bound of $v$ is already known, we next have

$$
\begin{aligned}
& \frac{1}{q} \frac{d}{d t} \int_{R^{n}}|u|^{q} \rho(x-y) d x \leq-\frac{4(q-1)}{q^{2}} \int_{R^{n}}\left|\nabla\left(|u|^{q / 2}\right)\right|^{2} \rho(x-y) d x \\
&+\frac{2}{q} \int_{R^{n}}|u|^{q / 2}\left|\nabla\left(|u|^{q / 2}\right)\right||\nabla \rho(x-y)| d x+M(v)\left(\int_{R^{n}}|u|^{2 q-2} \rho^{2-2 / q}(x-y) d x\right)^{1 / 2} \\
& \quad-a \int_{R^{n}}|u|^{q} \rho(x-y) d x+b \int_{R^{n}}|u|^{q-1} \rho(x-y) d x
\end{aligned}
$$

where

$$
M(v):=\alpha\left(\sup _{y \in R^{n}} \int_{R^{n}}|v|^{2} \rho^{2 / q}(x-y) d x\right)^{1 / 2} \leq \text { const. } \sup _{t \geq 0}\left(\sup _{y \in R^{n}} \int_{R^{n}}|v|^{2} \rho(x-y) d x\right)^{1 / 2} .
$$

REMARK 3.7. While the norms $\left(\int_{R^{n}}|v|^{2} \rho(x) d x\right)^{1 / 2}$ and $\left(\int_{R^{n}}|v|^{2} \rho^{2 / q}(x) d x\right)^{1 / 2}$ are obviously not equivalent for $\rho(x)=\left(1+|\varepsilon x|^{2}\right)^{-\nu}$, the expressions

$$
\sup _{y \in R^{n}}\left(\int_{R^{n}}|v|^{2} \rho^{2 / q}(x-y) d x\right)^{1 / 2} \text { and } \sup _{y \in R^{n}}\left(\int_{R^{n}}|v|^{2} \rho(x-y) d x\right)^{1 / 2}
$$

define equivalent norms in locally uniform space $L_{\mathrm{lu}}^{2}\left(R^{n}\right)$ provided that $\rho^{2 / q}$ and $\rho$ are both integrable (see Remark 4.1). Therefore, we take $\rho(x)=\left(1+|\varepsilon x|^{2}\right)^{-\nu}$ in (26) such that $\rho^{2 / q}$ is integrable, which is just a matter of choosing sufficiently large parameter $\nu$.

Let us next obtain suitable estimates of the terms appearing on the right hand side of (19). For the second term, using the estimate $|\nabla \rho(x-y)| \leq c_{0} \varepsilon \rho(x-y)$ (see (26)), we have

$$
\begin{aligned}
\frac{2}{q} \int_{R^{n}}|u|^{q / 2} & \left|\nabla\left(|u|^{q / 2}\right)\right||\nabla \rho(x-y)| d x \leq \frac{2}{q} c_{0} \varepsilon \int_{R^{n}}|u|^{q / 2}\left|\nabla\left(|u|^{q / 2}\right)\right| \rho(x-y) d x \\
& \leq \frac{1}{q} c_{0} \varepsilon \int_{R^{n}}|u|^{q} \rho(x-y) d x+\frac{1}{q} c_{0} \varepsilon \int_{R^{n}}\left|\nabla\left(|u|^{q / 2}\right)\right|^{2} \rho(x-y) d x .
\end{aligned}
$$

To estimate the third term we start with an auxiliary inequality valid for $n \leq 4$ :

$$
\begin{aligned}
& \left(\int_{R^{n}}|u|^{2 q-2} \rho^{2-2 / q}(x-y) d x\right)^{\frac{1}{2} \frac{q}{q-1}}=\left(\int_{R^{n}}\left[|u|^{q / 2} \rho^{1 / 2}(x-y)\right]^{\frac{4 q-4}{q}} d x\right)^{\frac{q}{2 q-2}} \\
& \quad=\left\||u|^{q / 2} \rho^{1 / 2}(\cdot-y)\right\|_{L^{(4 q-4) / q\left(R^{n}\right)}}^{2} \leq c\left\||u|^{q / 2} \rho^{1 / 2}(\cdot-y)\right\|_{H^{1}\left(R^{n}\right)}^{2} \\
& \quad=c\left(\left\||u|^{q / 2} \rho^{1 / 2}(\cdot-y)\right\|_{L^{2}\left(R^{n}\right)}^{2}+\left\|\left|\nabla\left[|u|^{q / 2} \rho^{1 / 2}(\cdot-y)\right]\right|\right\|_{L^{2}\left(R^{n}\right)}^{2}\right) \\
& \quad \leq c_{1}\left(\left\||u|^{q / 2} \rho^{1 / 2}(\cdot-y)\right\|_{L^{2}\left(R^{n}\right)}^{2}+\left\|\left|\nabla\left(|u|^{q / 2}\right)\right| \rho^{1 / 2}(\cdot-y)\right\|_{L^{2}\left(R^{n}\right)}^{2}\right) .
\end{aligned}
$$


Using (21) and the Young inequality, for the third term in (19) we get the bound

$$
\begin{aligned}
M(v) & \left(\int_{R^{n}}|u|^{2 q-2} \rho^{2-2 / q}(x-y) d x\right)^{1 / 2} \\
& \leq c(M(v), \bar{\delta})+\bar{\delta} c_{1} \int_{R^{n}}|u|^{q} \rho(x-y) d x+\bar{\delta} c_{1} \int_{R^{n}}\left|\nabla\left(|u|^{q / 2}\right)\right|^{2} \rho(x-y) d x
\end{aligned}
$$

For the fifth term we finally obtain

$$
b \int_{R^{n}}|u|^{q-1} \rho(x-y) d x \leq \tilde{\delta} \int_{R^{n}}|u|^{q} \rho(x-y) d x+C(\tilde{\delta}) \int_{R^{n}} \rho(x-y) d x .
$$

Connecting the estimates (19)-(23) and choosing $\varepsilon, \bar{\delta}, \tilde{\delta}$ small enough we arrive at the differential inequality

$$
\frac{d}{d t} \int_{R^{n}}|u|^{q} \rho(x-y) d x \leq-\frac{a q}{2} \int_{R^{n}}|u|^{q} \rho(x-y) d x+C\left(M(v),\|\rho\|_{L^{1}\left(R^{n}\right)}, b, q\right),
$$

which implies the required a priori bounds

$$
\sup _{t \geq 0} \sup _{\left\|\left(u_{0}, v_{0}\right)\right\|_{W_{1 \mathrm{u}}^{1, p}\left(R^{n}\right) \times L_{1 \mathrm{u}}^{2}\left(R^{n}\right)} \leq \lambda}\left\|u\left(t, u_{0}, v_{0}\right)\right\|_{L_{\mathrm{lu}}^{p z}\left(R^{n}\right)} \leq \lambda+\text { const. }
$$

and

$$
\limsup _{t \rightarrow+\infty} \sup _{\left\|\left(u_{0}, v_{0}\right)\right\|_{W_{\mathrm{lu}}^{1, p}\left(R^{n}\right) \times L_{\mathrm{lu}}^{2}\left(R^{n}\right)} \leq \lambda}\left\|u\left(t, u_{0}, v_{0}\right)\right\|_{L_{\mathrm{lu}}^{p z}\left(R^{n}\right)} \leq \text { const., }
$$

valid for any $\lambda>0$.

Step 3. Estimates obtained for $u$ in (24) and (25) give immediately corresponding $L_{\mathrm{lu}}^{p}\left(R^{n}\right)$ estimates for $v$, since it may be written in the integral form

$$
v\left(t, u_{0}, v_{0}\right)=v_{0} e^{-\delta t}+\int_{0}^{t} e^{-\delta(t-s)}\left(\beta u\left(s, u_{0}, v_{0}\right)+h\right) d s .
$$

We have thus estimated the solution $(u, v)$ in the norm of an auxiliary space $Y=$ $L_{\mathrm{lu}}^{p z}\left(R^{n}\right) \times L_{\mathrm{lu}}^{p}\left(R^{n}\right), p \in\left(n, p_{0}\right]$.

REMARK 3.8. By using the method described in Remark 2.2 this estimate is sufficient for validity of assumptions (ii) and (iii). Note that in the present example estimate (2) has the form

$$
\begin{array}{r}
\|F(u, v)\|_{L_{\mathrm{lu}}^{p}\left(R^{n}\right) \times L_{\mathrm{lu}}^{p}\left(R^{n}\right)}=\|f(u)-\alpha v\|_{L_{\mathrm{lu}}^{p}\left(R^{n}\right)}+\|\beta u+h\|_{L_{\mathrm{lu}}^{p}\left(R^{n}\right)} \\
\leq c\left\|\left(1+|u|^{z}\right)\right\|_{L_{\mathrm{lu}}^{p}\left(R^{n}\right)}+\alpha\|v\|_{L_{\mathrm{lu}}^{p}\left(R^{n}\right)}+\beta\|u\|_{L_{\mathrm{lu}}^{p}\left(R^{n}\right)}+\|h\|_{L_{\mathrm{lu}}^{p}\left(R^{n}\right)} \\
\leq \text { const. }\left(1+\|(u, v)\|_{L_{\mathrm{lu}}^{p z}\left(R^{n}\right) \times L_{\mathrm{lu}}^{p}\left(R^{n}\right)}+\|(u, v)\|_{L_{\mathrm{lu}}^{p z}\left(R^{n}\right) \times L_{\mathrm{lu}}^{p}\left(R^{n}\right)}^{z}\right) \\
=: g\left(\|(u, v)\|_{Y}\right) .
\end{array}
$$

Choose $Z=L_{\rho}^{p}\left(R^{n}\right) \times L_{\rho}^{p}\left(R^{n}\right), D_{Z}=W_{\rho}^{2, p}\left(R^{n}\right) \times L_{\rho}^{p}\left(R^{n}\right)$, recall that $n \leq 4, p \in\left(n, p_{0}\right]$, and observe that assumption (iv) is satisfied automatically. Also, thanks to the embedding

$$
X^{1 / 2}=W_{\mathrm{lu}}^{1, p}\left(R^{n}\right) \times L_{\mathrm{lu}}^{p}\left(R^{n}\right) \subset C_{\mathrm{bd}}^{0}\left(R^{n}\right) \times L_{\mathrm{lu}}^{p}\left(R^{n}\right), p>n,
$$


it is easy to verify condition $(\mathrm{v})$. Indeed, for any $B$ bounded in $X^{1 / 2}$ and each $\left(\phi_{1}, \psi_{1}\right)$, $\left(\phi_{2}, \psi_{2}\right) \in B$

$$
\begin{aligned}
\left\|F\left(\phi_{1}, \psi_{1}\right)-F\left(\phi_{2}, \psi_{2}\right)\right\|_{Z}=\left\|f\left(\phi_{1}\right)-f\left(\phi_{2}\right)-\alpha\left(\psi_{1}-\psi_{2}\right)\right\|_{L_{\rho}^{p}\left(R^{n}\right)} \\
+\beta\left\|\phi_{1}-\phi_{2}\right\|_{L_{\rho}^{p}\left(R^{n}\right)} \leq\left(L_{B}+\beta+\alpha\right)\left\|\left(\phi_{1}, \psi_{1}\right)-\left(\phi_{2}, \psi_{2}\right)\right\|_{Z}
\end{aligned}
$$

which proves the claim.

Finally, we need to check condition (vi) of Theorem 2.6. Thanks to the already verified assumption (iii) there is a bounded set $B_{0} \subset X^{1 / 2}$ absorbing bounded subsets of $X^{1 / 2}$. Also $B_{0}$ is bounded in $X^{1}$ and positively invariant.

Let $t_{n} \rightarrow \infty$ and $\left\{\left(u_{n}, v_{n}\right)\right\}$ be bounded in $X^{1 / 2}$. Verification of condition (vi) for the first coordinate $u$ is similar as in the previous example. For the second coordinate $v$ we will use its, slightly rearranged, integral representation

$$
\begin{aligned}
v\left(t, u_{0}, v_{0}\right)=\left[v_{0} e^{-\delta t}+\beta \int_{0}^{\tau} e^{-\delta(t-s)} u\left(s, u_{0}, v_{0}\right) d s\right. & \left.-\frac{e^{-\delta t}}{\delta} h\right] \\
& +\beta \int_{\tau}^{t} e^{-\delta(t-s)} u\left(s, u_{0}, v_{0}\right) d s+\frac{1}{\delta} h
\end{aligned}
$$

where $\tau$ is chosen such that $t_{n} \geq \tau$ for $n \geq n_{0}$ and

$$
\bigcup_{t \geq \tau}\left(u\left(t, u_{n}, v_{n}\right), v\left(t, u_{n}, v_{n}\right)\right) \subset B_{0} .
$$

Evidently, the square bracket decays to zero in $L_{\rho}^{p}\left(R^{n}\right)$ as $t=t_{n} \rightarrow \infty$. The subsequent integral term

$$
\beta \int_{\tau}^{t_{n}} e^{-\delta\left(t_{n}-s\right)} u\left(s, u_{n}, v_{n}\right) d s=: w_{n},
$$

is bounded in $W_{\text {lu }}^{2, p}\left(R^{n}\right)$ uniformly for $n \geq n_{0}$. Hence, as a consequence of Lemma 3.3, $\left\{w_{n}\right\}$ has a subsequence convergent in $L_{\rho}^{p}\left(R^{n}\right)$ to an element of $W_{\mathrm{lu}}^{1, p}\left(R^{n}\right)$. The proof of condition (vi) is thus complete allowing us to conclude that

Corollary 3.9. For $p \in\left(n, p_{0}\right]$ and $Z=L_{\rho}^{p}\left(R^{n}\right) \times L_{\rho}^{p}\left(R^{n}\right)$, the $C^{0}$-semigroup defined by $(12)$ on $X^{1 / 2}=W_{\mathrm{lu}}^{1, p}\left(R^{n}\right) \times L_{\mathrm{lu}}^{p}\left(R^{n}\right)$ possesses an $\left(X^{1 / 2}-Z\right)$-global attractor, which is bounded in $W_{\mathrm{lu}}^{2, p}\left(R^{n}\right) \times L_{\mathrm{lu}}^{p}\left(R^{n}\right)$.

4. Appendix. For convenience, throughout the paper we consider an integrable weight function $\rho$ of the form

$$
\rho(x)=\left(1+|\varepsilon x|^{2}\right)^{-\nu}, \nu>\frac{n}{2} .
$$

For $p \in(1, \infty)$ we define

$$
L_{\rho}^{p}\left(R^{n}\right)=\left\{\phi \in L_{\mathrm{loc}}^{p}\left(R^{n}\right):\|\phi\|_{L_{\rho}^{p}\left(R^{n}\right)}=\left(\int_{R^{n}}|\phi(x)|^{p} \rho(x) d x\right)^{1 / p}<\infty\right\} .
$$


Following [M-S], in applications we work in locally uniform spaces

$$
\begin{array}{r}
L_{\mathrm{lu}}^{p}\left(R^{n}\right)=\left\{\phi \in L_{\mathrm{loc}}^{p}\left(R^{n}\right):\|\phi\|_{L_{\mathrm{lu}}^{p}\left(R^{n}\right)=} \sup _{y \in R^{n}}\left(\int_{R^{n}}|\phi(x)|^{p} \tau_{y} \rho(x) d x\right)^{1 / p}<\infty\right. \\
\text { and } \left.\lim _{|z| \rightarrow 0}\left\|\tau_{z} \phi-\phi\right\|_{L_{\mathrm{lu}}^{p}\left(R^{n}\right)}=0\right\},
\end{array}
$$

where $\tau_{z} \rho(x)=\rho(x-z), x, z \in R^{n}$.

REMARK 4.1. Note that $L_{\mathrm{lu}}^{p}\left(R^{n}\right)$ contains both $L^{p}\left(R^{n}\right)$ and $L^{\infty}\left(R^{n}\right)$. Also,

$$
\sup _{y \in R^{n}}\left(\int_{\{|x-y|<1\}}|\phi(x)|^{p} d x\right)^{1 / p} \text { and } \sup _{y \in R^{n}}\left(\int_{R^{n}}|\phi(x)|^{p} \rho(x-y) d x\right)^{1 / p}
$$

define equivalent norms of $L_{\mathrm{lu}}^{p}\left(R^{n}\right)$ (see [Ca-Dl, Lemma 1]), which property is useful in many considerations. In particular, the definition of $L_{\mathrm{lu}}^{p}\left(R^{n}\right)$ is independent of the specific choice of the weight function $\rho$ inside an admissible class of such function (see e.g. [Ca-Dl] for details).

Based on the $L_{\text {lu }}^{p}\left(R^{n}\right)$ spaces one can introduce in a natural way Sobolev type locally uniform spaces $W_{\mathrm{lu}}^{k, p}\left(R^{n}\right)$ (see $[\mathrm{M}]$ ), containing all elements $\phi \in L_{\mathrm{lu}}^{p}\left(R^{n}\right)$ having distributional derivatives $D^{\sigma} \phi \in L_{\mathrm{lu}}^{p}\left(R^{n}\right),|\sigma| \leq k$, such that

$$
\|\phi\|_{W_{\mathrm{lu}}^{k, p}\left(R^{n}\right)}=\sum_{|\sigma| \leq k}\left\|D^{\sigma} \phi\right\|_{L_{\mathrm{lu}}^{p}\left(R^{n}\right)}<\infty .
$$

As shown in [Ch-Dl1] the set $C_{\mathrm{bd}}^{k}\left(R^{n}\right)$, consisting of functions having bounded derivatives up to the order $k$, is dense in $W_{\mathrm{lu}}^{k, p}\left(R^{n}\right)$ for each $p \in(1, \infty)$.

We prove below an embedding of locally uniform spaces into Hölder type spaces. Our target space will be $C^{\mu}\left(R^{n}\right)$, which consists of functions $\phi \in C_{\mathrm{bd}}^{0}\left(R^{n}\right)$ being uniformly Hölder continuous in $R^{n}$ and normed by

$$
\|\phi\|_{C^{\mu}\left(R^{n}\right)}=\sup _{x \in R^{n}}|\phi(x)|+\sup _{x, y \in R^{n}, x \neq y} \frac{|\phi(x)-\phi(y)|}{|x-y|^{\mu}},
$$

(see [T, p. 67], where such a space is denoted by $B^{\mu}\left(R^{n}\right)$ ).

Lemma 4.2. Let $0<\mu<1-\frac{n}{p}$ and $\rho: R^{n} \rightarrow(0, \infty)$ be given by $(26)$. Then

$$
W_{\mathrm{lu}}^{1, p}\left(R^{n}\right) \subset C^{\mu}\left(R^{n}\right) .
$$

ProOF. For $y, z \in R^{n}, 0<|z| \leq 1$, we have

$$
\begin{aligned}
\frac{|\phi(y+z)-\phi(y)|}{|z|^{\mu}} \leq \sup _{|z| \leq 1, z \neq 0} \frac{\left|\tau_{-y} \phi(z)-\tau_{-y} \phi(0)\right|}{|z|^{\mu}} \\
\leq c\left\|\tau_{-y} \phi\right\|_{W^{1, p}(\{|x|<1\})}=c\|\phi\|_{W^{1, p}(\{|x-y|<1\})}
\end{aligned}
$$

so that substituting $z=x-y$ we obtain

$$
\sup _{|x-y| \leq 1, x \neq y} \frac{|\phi(x)-\phi(y)|}{|x-y|^{\mu}} \leq\|\phi\|_{W_{\mathrm{lu}}^{1, p}\left(R^{n}\right)}
$$


(see Remark 4.1). Using next the embedding $W_{\mathrm{lu}}^{1, p}\left(R^{n}\right) \subset C_{\mathrm{bd}}^{0}\left(R^{n}\right)$ (see [Ch-Dl1, Corollary 1]), we observe that

$$
\begin{aligned}
& \|\phi\|_{C^{\mu}\left(R^{n}\right)=} \sup _{x \in R^{n}}|\phi(x)|+\sup _{x, y \in R^{n}, x \neq y} \frac{|\phi(x)-\phi(y)|}{|x-y|^{\mu}} \\
& \quad \leq 3 \sup _{x \in R^{n}}|\phi(x)|+\sup _{|x-y| \leq 1, x \neq y} \frac{|\phi(x)-\phi(y)|}{|x-y|^{\mu}} \leq C\|\phi\|_{W_{1 \mathrm{u}}^{1, p}\left(R^{n}\right)},
\end{aligned}
$$

where in the last estimate we decompose $R^{n}=\{y:|x-y| \leq 1\} \cup\{y:|x-y|>1\}$. The proof is complete.

\section{References}

[Ad] R. A. Adams, Sobolev Spaces, Academic Press, New York, 1975.

[B-V] A. V. Babin, M. I. Vishik, Attractors of Evolution Equations, North-Holland, Amsterdam, 1992.

[Ca-Dl] A. N. Carvalho, T. DŁotko, Partially dissipative systems in uniformly local spaces, submitted.

[Ch-Dl] J. W. Cholewa, T. DŁotko, Global Attractors in Abstract Parabolic Problems, Cambridge University Press, Cambridge, 2000.

[Ch-Dl1] J. W. Chоlewa, T. DŁotKo, Cauchy problems in weighted Lebesgue spaces, Czechoslovak Math. J. (to appear).

[F] E. FEIREISL, Bounded, locally compact global attractors for semilinear damped wave equations in $R^{n}$, Differential Integral Equations 9 (1996), 1147-1156.

[Ha] J. K. Hale, Asymptotic Behavior of Dissipative Systems, Amer. Math. Soc., Providence, 1988.

[He] D. Henry, Geometric Theory of Semilinear Parabolic Equations, Springer, Berlin, 1981.

[La] O. Ladyzhenskaya, Attractors for Semigroups and Evolution Equations, Cambridge University Press, Cambridge, 1991.

[M] A. Mielke, The complex Ginzburg-Landau equation on large and unbounded domains: sharper bounds and attractors, Nonlinearity 10 (1997), 199-222.

[M-S] A. Mielke, G. Schneider, Attractors for modulation equations on unbounded domains-existence and comparison, Nonlinearity 8 (1995), 743-768.

[T] H. Tanabe, Functional Analytic Methods for Partial Differential Equations, Dekker, New York, 1997. 